\title{
Methicillin-resistant Staphylococcal periprosthetic joint infections can be effectively controlled by systemic and local daptomycin
}

Feng-Chih Kuo ${ }^{1,2^{*}}$, Shih-Hsiang Yen ${ }^{1,2}$, Kuo-Ti Peng ${ }^{3}$, Jun-Wen Wang ${ }^{1,2}$ and Mel S. Lee ${ }^{1,2}$

\begin{abstract}
Background: Methicillin-resistant Staphylococcus remains a serious problem in the treatment of periprosthetic joint infection (PJI). Higher failure rates were reported when vancomycin was used in 2-stage exchange arthroplasty. Therefore a better therapeutic drug is needed to treat PJI caused by methicillin-resistant organisms. The purpose of the study was to evaluate the safety and efficacy of daptomycin when administered in bone cement combined with systemic use for methicillin-resistant Staphylococci PJl.
\end{abstract}

Methods: We conducted a retrospective study from January 2010 to December 2012. Twenty-two patients (10 knees and 12 hips) with PJl caused by methicillin-resistant Staphylococcus species underwent 2-stage revision arthroplasty. In the first stage, $10 \%$ daptomycin (weight daptomycin per weight bone cement) was incorporated into polymethylmethacrylate bone cement, and systemic daptomycin $(6 \mathrm{mg} / \mathrm{kg})$ was administered postoperatively for 14 days. In the second stage, $2.5 \% \mathrm{w} / \mathrm{w}$ daptomycin was used in the bone cement. The minimum follow-up was 2 years or until recurrence of infection.

Results: The infecting organisms included methicillin-resistant Staphylococcus aureus in 10 patients, methicillin-resistant Staphylococcus epidermidis in 8 patients and methicillin-resistant coagulase-negative Staphylococci in 4 patients. The mean follow-up duration was 33.7 months (range, 24-51 months). The treatment success rate was $100 \%$. Only one patient developed asymptomatic transient elevation of the creatine phosphokinase level. No patient experienced any adverse effects related to daptomycin such as myositis, rhabdomyolysis, peripheral neuropathy, derangement of liver function, or eosinophilic pneumonia.

Conclusions: In this series, no serious adverse events occurred. Our protocol, using daptomycin-impregnated cement combined with short duration of systemic daptomycin, appears to be an effective and safe treatment for methicillin-resistant Staphylococcus PJ.

Keyword: Daptomycin, Periprosthetic joint infection, Cement, Staphylococcus

\footnotetext{
* Correspondence: fongchikuo@cgmh.org.tw

${ }^{1}$ Department of Orthopaedic Surgery, Kaohsiung Chang Gung Memorial

Hospital, No 123, Ta Pei Road, Niao Sung Dist, Kaohsiung, Taiwan

${ }^{2}$ Chang Gung University College of Medicine, Kaohsiung, Taiwan

Full list of author information is available at the end of the article
} 


\section{Background}

Periprosthetic joint infections (PJI) of the hip and knee are disastrous complications that occur in approximately 1 to $2 \%$ of patients after total joint arthroplasties [1]. The management of PJI may require long-term antibiotic suppression, surgical debridement, one-stage or 2stage revision, resection arthroplasty, arthrodesis, or amputation. The "gold standard" treatment for chronic PJI in North America is 2-stage revision [2]. The procedure consists of removal of the infected prosthesis in the first stage, followed by replacing it with a high-dose antibiotic cement spacer to eradicate the infection and prevent joint space contracture between stages [3]. Once the infection has been treated with systemic antibiotics, the second stage is performed to implant a new prosthesis.

Most PJI are caused by Gram-positive cocci, including Staphylococcus species [4]. Methicillin-resistant organisms account for up to $74 \%$ of PJI in some reports [5]. Vancomycin is most commonly incorporated into polymethylmethacrylate (PMMA) bone cement and subsequently used intravenously for the treatment of methicillinresistant Staphylococcus aureus (MRSA) [6]. The successful clinical control of chronic PJI due to methicillinresistant organisms varies from 48 to $89 \%[7,8]$ in the hip and 60 to $74 \%[9,10]$ in the knee when vancomycin is used in 2-stage exchange arthroplasty. These results have led orthopedic surgeons to seek new therapeutic strategies for PII caused by methicillin-resistant Staphylococcus spp.

Daptomycin is a novel cyclic lipopeptide antibiotic secreted by Streptomyces roseosporus. Daptomycin has excellent activity against Gram-positive bacteria through disruption of multiple bacterial plasma membrane functions, without penetrating the cytoplasm [11]. Clinical experience with daptomycin treatment of PJI is limited to systemic intravenous use in small case series [12-14]. Several in vitro studies also showed that daptomycin could be locally delivered from PMMA bone cement without impairing cement strength $[15,16]$. Only one clinical report showed combined use of daptomycin in bone cement and intravenously to treat chronic PJI in a 2-stage surgery [17].

The aim of this study was to review the results of daptomycin used in PMMA bone cement and systemically in 2-stage exchange surgeries for the treatment of PJI due to methicillin-resistant Staphylococcus species.

\section{Methods}

\section{Study design and setting}

This retrospective, descriptive study was conducted from January 2010 to December 2012 in a 2700-bed medical center. Consecutive adults who underwent 2stage revision arthroplasty using local and systemic daptomycin for the treatment of PJI caused by methicillinresistant Staphylococcus species were included. The use of local and systemic daptomycin for the treatment of methicillin-resistant Staphylococcus PJI was just done during the study period. The exclusion criteria were PJI caused by methicillin-sensitive Staphylococcus species, Gram-negative bacteria, Mycobacterium tuberculosis, fungi and polymicrobial infections. Diagnosis was confirmed by isolation of methicillin-resistant bacteria in at least 2 intraoperative cultures. All clinical data were collected retrospectively by reviewing electronic medical records. The Institutional Review Board of the Chang Gung Memorial Hospital Foundation approved the study (IRB No. 103-3637B).

\section{Surgical procedures and postoperative care}

Patients with early prosthesis infections and acute haematogenous infections with failure of DAIR surgery (debridement, antibiotics, irrigation and prosthesis retention), or with prior implant loosening and late chronic infections, underwent two-stage revision surgery [18]. The infecting organisms included MRSA in 10 patients, methicillin-resistant Staphylococcus epidermidis (MRSE) in 8 patients and methicillin-resistant coagulase-negative Staphylococci (MRCoNS) in 4 patients. The minimum inhibitory concentrations (MICs) of vancomycin were determined as $>1.5 \mathrm{mg} / \mathrm{L}$ in all patients. Higher vancomycin MICs (>1.5 mg/L) have a higher risk of treatment failure for MRSA treated with vancomycin [19]. In the first stage, the operative procedure included removal of the implants, aggressive debridement of the joint and insertion of a high-dose, daptomycin-loaded cement spacer or beads. Twenty (91\%) of the 22 patients were treated with bead fashion of antibiotic-loaded cement. Erythrocyte sedimentation rate (ESR), C-reactive protein $(\mathrm{CRP})$, complete blood count $(\mathrm{CBC})$ with differential and creatine phosphokinase $(\mathrm{CPK})$ were monitored weekly postoperatively. In the second stage, new prostheses were reimplanted and low-dose daptomycin-loaded cement was used if cementing fixation was needed. No patients had a joint aspiration before reimplantation. The criteria for reimplantation included a reduced ESR, return to near normal CRP, and satisfactory wound status. A closed suction drain inserted immediately after each surgery was removed 2-5 days later when the amount of daily drainage was less than $60 \mathrm{~mL}$ per day. The postoperative course of periprosthetic knee infection treatment consisted of a 3-day period of immobilization in a hingeknee brace, followed by gradually continuous passive motion exercise, and protected weight-bearing activity. All the revision total hip arthroplasty were posterior approach. The postoperative course of periprosthetic hip infection treatment consisted of a 3-day period of immobilization using skin traction $(2 \mathrm{~kg})$ followed by protected weight-bearing activity under hip abduction and brace protection to prevent dislocation. 


\section{Composition of bone cement}

If the methicillin-resistant microorganism had been identified at the time of resection arthroplasty, the dose of daptomycin was $4 \mathrm{~g}$ per $40 \mathrm{~g}$ package of bone cement (Stryker Orthopaedics, Mahwah, New Jersey) to reach therapeutic levels in the joint fluid [20]. If the infecting microorganism could not be identified preoperatively, a combination of $4 \mathrm{~g}$ of daptomycin and $4 \mathrm{~g}$ of ceftazidime (10\% w/w for each antibiotic) per $40 \mathrm{~g}$ package of bone cement was used. In the second stage, the antibiotic bone cement spacer or beads were carefully removed. Intraoperative tissue samples were also taken for culture, as in the first stage. All reimplantations were performed after a 2-week antibiotic holiday without elevation of ESR and CRP. After prophylaxis with intravenous $1 \mathrm{~g}$ vancomycin, the patients underwent prosthesis reimplantation with $1 \mathrm{~g}$ daptomycin $(2.5 \% \mathrm{w} / \mathrm{w})$ in a pack of $40 \mathrm{~g}$ bone cement [Stryker Orthopaedics, Mahwah, New Jersey] without decreasing the cement strength $[15,16]$ for knee or hip prosthesis fixation. For knee implantation, we usually use $1 \mathrm{~g}$ daptomycin in a pack of $40 \mathrm{~g}$ bone cement for tibia and patellar component fixation and $1 \mathrm{~g}$ vancomycin in another pack of bone cement for femoral component fixation. For hip reimplantation, three of 12 patients used $1 \mathrm{~g}$ daptomycin in a pack of $40 \mathrm{~g}$ bone cement for cup fixation.

\section{Microbiological investigation}

During the first stage of the 2-stage procedure, at least 3 samples of periprosthetic tissue (synovial membranes or bone) were obtained for cultures under aerobic and anaerobic conditions. One or 2 synovial fluid samples were put into blood culture flasks to increase sensitivity for the diagnosis of PJI [21]. All the samples of synovial fluid or periprosthetic tissue were incubated for at least 14 days [22]. These patients received 14 days of intravenous daptomycin $6 \mathrm{mg} / \mathrm{kg} /$ day postoperatively.

\section{Definition of outcome}

Two-stage reimplantation was defined as being successful if the patient had no symptom or sign of infection (no pain, swelling, erythema, warmth, wound discharge, loosening of the prosthesis), had normal CRP and ESR, and did not require reoperation (including irrigation and debridement with prosthesis retention and repeat resection) after a 2-year follow-up [23]. Treatment failure was defined as: a) death related to the infection; b) recurrence of infectious symptoms and signs; c) requiring a reoperation.

\section{Statistical analysis}

Categorical variables were expressed as percentages and numerical data as medians and ranges. Infection control rate was calculated for patients treated with daptomycin. All patients were included in the analysis.

\section{Results}

Seventy-six patients had PJI of the knee and hip during the study period. Twenty-two (10 knees, 12 hips) had PJI caused by methicillin-resistant Staphylococcus species and underwent 2-stage revision arthroplasty, with daptomycin used in PMMA bone cement and systemically (Table 1). There were 16 men and 6 women. Two patients had Staphylococcus aureus bacteremia at first presentation. The average age at the time of the 2-stage revision was 64.4 years (range, 38-87 years). The mean Charlson comorbidity index was 3.95 (range, 2-6). The surgical procedures before enrolment included primary hip arthroplasty (10 patients), primary knee arthroplasty (8), revision total hip arthroplasty (2) and revision total knee arthroplasty (2). Fourteen subjects underwent debridement with prosthesis retention before the 2-stage revision. The mean interval between the previous surgery and the first stage of the two-stage revision was 32 (8-120) months. The mean interim period between the two-stage debridement and reimplantation was averaged 14 weeks (range, 10-18 weeks). There was no breakage of the cement spacer during the interim. The mean follow-up duration was 33.7 months (range, 24-57 months). No patient was lost to follow-up. The treatment success rate was $100 \%$ without recurrence of infection. One patient developed asymptomatic transient elevation of the CPK level. No adverse effect related to daptomycin, such as myositis, rhabdomyolysis, peripheral neuropathy, derangement of liver function or eosinophilic pneumonia was observed in our study.

\section{Discussion}

Methicillin-resistant Staphylococci remain a challenge because the current protocol often has inferior results compared with protocols used with other organisms for the treatment of PJI [8, 24, 25]. The 2-stage protocol for resistant organisms has had success rates ranging between 48 and $89 \%[7-10,24,25]$, while the average success rate for less virulent organisms was $85 \%$ $95 \%$ $[3,8]$. Most protocols used vancomycin and an aminoglycoside in PMMA bone cement combined with systemic vancomycin for $2 \sim 6$ weeks after first-stage resection arthroplasty $[8-10,26]$. To our knowledge, this study was the first case-series report of daptomycin used in PMMA bone cement to treat PII caused solely by methicillin-resistant Staphylococcus species. Using $4 \mathrm{gm}$ daptomycin in 40-g PMMA bone cement combined with subsequent systemic use for 14 days in the first stage, followed by $1 \mathrm{~g}$ daptomycin in 40-g PMMA bone cement in the second stage, we achieved a $100 \%$ infection control rate with a mean follow-up of 2.8 years. We 
Table 1 Characteristics of patients with periprosthetic joint infections caused by methicillin-resistant Staphylococcus undergoing 2-stage revision arthroplasties

\begin{tabular}{|c|c|c|c|c|c|c|c|c|c|}
\hline No. & Sex & Age & Surgical procedures & $\mathrm{CCl}$ & Pathogen & $\begin{array}{l}\text { Antibiotic regimen (bone } \\
\text { cement } \mathrm{g} / \mathrm{systemic} \mathrm{mg} / \mathrm{kg} \text { ) }\end{array}$ & $\begin{array}{l}\text { Use daptomycin in bone } \\
\text { cement at reimplantation (Y/N) }\end{array}$ & FU (months) & Outcome \\
\hline 1 & $M$ & 65 & DAIR for TKA & 4 & MRSA & DAP 4/DAP 6 & Y & 26 & Infection controlled \\
\hline 2 & M & 51 & THA & 4 & MRSA & DAP $4+$ CEF 4/DAP 6 & $N$ & 32 & Infection controlled \\
\hline 3 & M & 72 & DAIR for revision TKA & 5 & MRSE & DAP 4/DAP 6 & Y & 43 & Infection controlled \\
\hline 4 & M & 53 & Revision THA & 2 & MRCoNS & DAP $4+$ CEF 4/DAP 6 & $N$ & 42 & Infection controlled \\
\hline 5 & $\mathrm{~F}$ & 60 & TKA & 3 & MRSA & DAP $4+$ CEF 4/DAP 6 & Y & 38 & Infection controlled \\
\hline 6 & $\mathrm{~F}$ & 80 & DAIR for TKA & 5 & MRSE & DAP 4/DAP 6 & Y & 26 & Infection controlled \\
\hline 7 & M & 66 & DAIR for THA & 3 & MRCoNS & DAP 4/DAP 6 & $N$ & 51 & Infection controlled \\
\hline 8 & M & 44 & DAIR for THA & 2 & MRSE & DAP 4/DAP 6 & $\mathrm{~N}$ & 42 & Infection controlled \\
\hline 9 & M & 74 & DAIR for TKA & 4 & MRSE & DAP 4/DAP 6 & Y & 25 & Infection controlled \\
\hline 10 & M & 45 & DAIR for THA & 3 & MRSE & DAP 4/DAP 6 & $N$ & 26 & Infection controlled \\
\hline 11 & M & 51 & DAIR for THA & 4 & MRSA & DAP 4/DAP 6 & Y & 44 & Infection controlled \\
\hline 12 & $\mathrm{~F}$ & 67 & TKA & 5 & MRSA & DAP $4+$ CEF 4/DAP 6 & Y & 32 & Infection controlled \\
\hline 13 & $\mathrm{~F}$ & 86 & TKA & 5 & MRSA & DAP $4+$ CEF 4/DAP 6 & Y & 37 & Infection controlled \\
\hline 14 & M & 51 & DAIR for THA & 5 & MRSA & DAP 4/DAP 6 & $\mathrm{~N}$ & 40 & Infection controlled \\
\hline 15 & $\mathrm{~F}$ & 86 & TKA & 5 & MRSA & DAP $4+$ CEF 4/DAP 6 & Y & 30 & Infection controlled \\
\hline 16 & $\mathrm{~F}$ & 81 & THA & 3 & MRSA & DAP $4+$ CEF 4/DAP 6 & Y & 32 & Infection controlled \\
\hline 17 & M & 81 & DAIR for revision TKA & 5 & MRSE & DAP 4/DAP 6 & Y & 30 & Infection controlled \\
\hline 18 & M & 51 & DAIR for revision THA & 4 & MRCoNS & DAP 4/DAP 6 & $\mathrm{~N}$ & 31 & Infection controlled \\
\hline 19 & M & 50 & DAIR for THA & 2 & MRSE & DAP 4/DAP 6 & $\mathrm{~N}$ & 27 & Infection controlled \\
\hline 20 & M & 87 & TKA & 6 & MRSA & DAP $4+$ CEF 4/DAP 6 & Y & 24 & Infection controlled \\
\hline 21 & M & 38 & DAIR for THA & 2 & MRSE & DAP 4/DAP 6 & $\mathrm{~N}$ & 30 & Infection controlled \\
\hline 22 & M & 78 & DAIR for revision THA & 6 & MRCONS & DAP 4/DAP 6 & Y & 33 & Infection controlled \\
\hline
\end{tabular}

$D A I R$ debridement, antibiotics, irrigation and prosthesis retention, $C C I$ Charlson comorbidity index, DM diabetes mellitus, HTN hypertension, CVA cerebral vascular accident, $T K A$ total knee arthroplasty, $L C$ liver cirrhosis, $R A$ rheumatoid arthritis, $T H A$ total hip arthroplasty, $C A D$ coronary artery disease, $B M I$ body mass index, $H B V$ hepatic $B$ virus, $H C V$ hepatic $C$ virus, CKD chronic kidney disease, COPD chronic obstructive pulmonary disease, ESRD end-stage renal disease, DAP daptomycin, CEF ceftazidime, MRSA methicillin-resistant Staphylococcus aureus, MRCoNS methicillin-resistant coagulase-negative Staphylococci, MRSE methicillin-resistant

Staphylococcus epidermis, FU follow-up

attributed the results to the readiness of daptomycin release from the PMMA bone cement and its excellent bactericidal effect against methicillin-resistant strains. Hall et al. showed that daptomycin could be released from PMMA cement at a rate similar to that of vancomycin in vitro study [27]. These results suggested that local concentrations of daptomycin from daptomycinloaded PMMA cement were above the MIC value for most Gram-positive cocci. The efficacy of daptomycin against Staphylococci, compared to vancomycin, has been demonstrated in vivo and in vitro studies. Daptomycin had a higher bactericidal rate than vancomycin (92\% vs. $70 \%$ ) in an in vitro study [28]. Daptomycin showed greater and more rapid bactericidal activity than vancomycin in mice infected by MRSA [29]. Systemic daptomycin also had a higher success rate than vancomycin in 2-stage revision arthroplasty in a randomized controlled trial [14].

The actual dosage of daptomycin added in the bone cement as a spacer to treat PJI is unclear. Cortes et al. reported the first case report of use of daptomycin $10 \mathrm{~g}$ and gentamicin $10 \mathrm{~g}$ in the bone cement (each agent at $5 \% \mathrm{w} / \mathrm{w}$ ) in two-stage revision hip surgery for prosthetic joint infection [17]. In an international consensus meeting on periprosthetic joint infection, $2 \mathrm{~g}$ daptomycin $(5 \% \mathrm{w} / \mathrm{w})$ was recommended in spacers [30]. Rouse et al. found that $3 \mathrm{~g}$ daptomycin $(7.5 \% \mathrm{w} / \mathrm{w})$ did not decrease the tensile or compressive strength of PMMA bone cement and retained biologic activity after PMMA cement polymerization in an in vivo rat model [31]. According to an in vitro study, the mean percentage of daptomycin elution increased with an increase in daptomycin loading $[2.5,7.5$, and $15.0 \% \mathrm{w} / \mathrm{w}]$ in PMMA bone cement. Therefore, we thought higher dose daptomycin $(10 \% \mathrm{w} / \mathrm{w})$ in PMMA bone cement to reach local therapeutic levels for the treatment of PJI due to methicillin-resistant Staphylococcus species.

The safety of systemic use of daptomycin after resection arthroplasty is also a concern. Byren et al. [14] used daptomycin at 6 or $8 \mathrm{mg} / \mathrm{kg}$ for 6 weeks after prosthesis 
removal in a randomized trial. They found $8 \%$ ( 2 of 25 ) adverse events (AEs) in the $6-\mathrm{mg} / \mathrm{kg}$ group and $16.7 \%$ (4 of 24) AEs in the $8-\mathrm{mg} / \mathrm{kg}$ group. The AEs included skin rash, rhabdomyolysis and increase CPK. In another study, severe side effects (one case of acute renal failure due to massive rhabdomyolysis, one of eosinophilic pneumonia and 2 cases of asymptomatic transient CPK level elevation) were also reported with daptomycin at a dose of $6.6 \mathrm{mg} / \mathrm{kg} /$ day for an average of 44.9 days in the treatment of PJI [13]. Our group underwent 2-week systemic antibiotic therapy after first-stage surgery without obviously poorer results than in other reports [32]. By decreasing the duration of systemic daptomycin use, 21 of 22 patients tolerated the treatment. No patients developed gastrointestinal or food intolerance. Only one patient developed asymptomatic transient elevation of the CPK level. No patient experienced any severe adverse effects related to daptomycin. Daptomycin also possesses clinical and practical advantage over vancomycin, like once daily versus twice daily dosing, less therapeutic drug monitoring and potential cost savings. We thought a shorter course of systemic use of daptomycin would be advisable because the adverse events would be fewer and the infection control rates would not be compromised.

In this study, we had used prophylactic intravenous vancomycin combined with local daptomycin for the treatment of MRSA PJI at reimplantaiton stage. Vancomycin has been known as the drug of choice to prevent MRSA PJI in primary or reimplantation arthroplasties following MRSA infection [33, 34]. We did not choose intravenous daptomycin for 2 reasons. First, we would like to compare this result with our previous experience of using systemic and local vancomycin by changing the topical antibiotic regime only. This could reduce the confounding effect if systemic daptomycin were used. Second, our infection control policy precluded us to supersede the first-line vancomycin to the second-line daptomycin for systemic use without drug sensitivity test and minimal inhibition concentration test.

The study has limitations. First, it was a retrospective design, and we could not know what proportion of patients would fail the two-stage protocol if vancomycin were used. The evaluation of a prospective cohort comparing daptomycin to vancomycin may be warranted in the future. Second, the sample size was small, making it difficult to obtain statistically significant results. Third, the optimal ratio of daptomycin to PMMA cement in vivo study was unknown, and we did not check the daptomycin level in the joint fluid. The strength of our study is that it offered clinical data from a cohort of patients, and reported the safe use of daptomycin in PMMA cement and intravenously in 2-stage revision surgery. But further studies are required to evaluate the long-term outcomes.

\section{Conclusions}

In conclusion, daptomycin-impregnated cement combined with a short duration of systemic daptomycin appears to be an effective treatment for methicillin-resistant Staphylococci PJI. The protocol could lessen the AEs related to daptomycin and provided satisfactory infection control rates.

\section{Competing interests \\ The authors declare that they have no competing interests.}

\section{Authors' contributions}

FCK established the clinical and the clinical database, conceived and designed the study and wrote the draft of manuscript. SHY conducted the study. KTP performed the analyses. FCK wrote the first draft of the manuscript. JWW and MSL performed critical revision the final version of the manuscript for important intellectual content, and final approval. All authors read and approved the final manuscript.

\section{Acknowledgement \\ None.}

\section{Author details}

${ }^{1}$ Department of Orthopaedic Surgery, Kaohsiung Chang Gung Memorial Hospital, No 123, Ta Pei Road, Niao Sung Dist, Kaohsiung, Taiwan. ${ }^{2}$ Chang Gung University College of Medicine, Kaohsiung, Taiwan. ${ }^{3}$ Department of Orthopaedic Surgery, Chiayi Chang Gung Memorial Hospital, Chiayi, Taiwan.

Received: 13 July 2015 Accepted: 22 January 2016

Published online: 01 February 2016

\section{References}

1. Adeli B, Parvizi J. Strategies for the prevention of periprosthetic joint infection. J Bone Joint Surg (Br). 2012;94(11 Suppl A):42-6.

2. Ibrahim MS, Raja S, Khan MA, Haddad FS. A multidisciplinary team approach to two-stage revision for the infected hip replacement: a minimum five-year follow-up study. Bone joint J. 2014;96-B(10):1312-8.

3. Toulson C, Walcott-Sapp S, Hur J, Salvati E, Bostrom M, Brause B, et al. Treatment of infected total hip arthroplasty with a 2-stage reimplantation protocol: update on "our institution's" experience from 1989 to 2003. J Arthroplasty. 2009;24(7):1051-60.

4. Davis JS. Management of bone and joint infections due to Staphylococcus aureus. Intern Med J. 2005;35 Suppl 2:S79-96.

5. Moran GJ, Krishnadasan A, Gorwitz RJ, Fosheim GE, McDougal LK, Carey RB, et al. Methicillin-resistant $\mathrm{S}$. aureus infections among patients in the emergency department. N Engl J Med. 2006;355(7):666-74.

6. Kuzyk PR, Dhotar HS, Sternheim A, Gross AE, Safir O, Backstein D. Two-stage revision arthroplasty for management of chronic periprosthetic hip and knee infection: techniques, controversies, and outcomes. J Am Acad Orthop Surg. 2014;22(3):153-64.

7. Kilgus DJ, Howe DJ, Strang A. Results of periprosthetic hip and knee infections caused by resistant bacteria. Clin Orthop Relat Res. 2002;404:116-24.

8. Volin SJ, Hinrichs SH, Garvin KL. Two-stage reimplantation of total joint infections: a comparison of resistant and non-resistant organisms. Clin Orthop Relat Res. 2004:427:94-100.

9. Parvizi J, Azzam K, Ghanem E, Austin MS, Rothman RH. Periprosthetic infection due to resistant staphylococci: serious problems on the horizon. Clin Orthop Relat Res. 2009;467(7):1732-9.

10. Mittal Y, Fehring TK, Hanssen A, Marculescu C, Odum SM, Osmon D. Twostage reimplantation for periprosthetic knee infection involving resistant organisms. J Bone Joint Surg Am. 2007:89(6):1227-31.

11. Woodworth JR, Nyhart Jr EH, Brier GL, Wolny JD, Black HR. Single-dose pharmacokinetics and antibacterial activity of daptomycin, a new lipopeptide antibiotic, in healthy volunteers. Antimicrob Agents Chemother. 1992;36(2):318-25

12. Rao N, Regalla DM. Uncertain efficacy of daptomycin for prosthetic joint infections: a prospective case series. Clin Orthop Relat Res. 2006;451:34-7.

13. Corona Pérez-Cardona PS, Barro Ojeda V, Rodriguez Pardo D, Pigrau Serrallach C, Guerra Farfán E, Amat Mateu C, et al. Clinical experience with daptomycin 
for the treatment of patients with knee and hip periprosthetic joint infections. J Antimicrob Chemother. 2012;67(7):1749-54.

14. Byren I, Rege S, Campanaro E, Yankelev S, Anastasiou D, Kuropatkin G, et al. Randomized controlled trial of the safety and efficacy of Daptomycin versus standard-of-care therapy for management of patients with osteomyelitis associated with prosthetic devices undergoing two-stage revision arthroplasty. Antimicrob Agents Chemother. 2012;56(11):5626-32.

15. Hsu YM, Liao CH, Wei YH, Fang HW, Hou HH, Chen CC, et al. Daptomycinloaded polymethylmethacrylate bone cement for joint arthroplasty surgery. Artif Organs. 2014;38(6):484-92.

16. Lewis G, Brooks JL, Courtney HS, Li Y, Haggard WO. An Approach for determining antibiotic loading for a physician-directed antibiotic-loaded PMMA bone cement formulation. Clin Orthop Relat Res. 2010;468(8):2092-100.

17. Cortes NJ, Lloyd JM, Koziol L, O'Hara L. Successful clinical use of daptomycinimpregnated bone cement in two-stage revision hip surgery for prosthetic joint infection. Ann Pharmacother. 2013;47(1):e2.

18. Tsukayama DT, Estrada R, Gustilo RB. Infection after total hip arthroplasty. A study of the treatment of one hundred and six infections. J Bone Joint Surg Am. 1996;78(4):512-23.

19. Lodise TP, Graves J, Evans A, Graffunder E, Helmecke M, Lomaestro BM, et al. Relationship between vancomycin MIC and failure among patients with methicillin-resistant Staphylococcus aureus bacteremia treated with vancomycin. Antimicrob Agents Chemother. 2008;52(9):3315-20.

20. Penner MJ, Masri BA, Duncan CP. Elution characteristics of vancomycin and tobramycin combined in acrylic bone-cement. J Arthroplasty. 1996;11(8):939-44.

21. Font-Vizcarra L, Garcia S, Martinez-Pastor JC, Sierra JM, Soriano A. Blood culture flasks for culturing synovial fluid in prosthetic joint infections. Clin Orthop Relat Res. 2010;468(8):2238-43.

22. Fink B, Makowiak C, Fuerst M, Berger I, Schäfer P, Frommelt L. The value of synovial biopsy, joint aspiration and C-reactive protein in the diagnosis of late peri-prosthetic infection of total knee replacements. J Bone Joint Surg (Br). 2008;90(7):874-8.

23. Parvizi J, Gehrke T, Chen AF. Proceedings of the International Consensus on Periprosthetic Joint Infection. Bone Joint J. 2013;95-B(11):1450-2.

24. Parry MC, Duncan CP. The challenge of methicillin resistant staphylococcal infection after total hip replacement: overlooked or overstated? Bone Joint J. 2014;96-B(11 Supple A):60-5.

25. Leung F, Richards CJ, Garbuz DS, Masri BA, Duncan CP. Two-stage total hip arthroplasty: how often does it control methicillin-resistant infection? Clin Orthop Relat Res. 2011;469(4):1009-15.

26. Salgado CD, Dash S, Cantey JR, Marculescu CE. Higher risk of failure of methicillin-resistant Staphylococcus aureus prosthetic joint infections. Clin Orthop Relat Res. 2007:461:48-53.

27. Hall EW, Rouse MS, Jacofsky DJ, Osmon DR, Hanssen AD, Steckelberg JM, et al. Release of daptomycin from polymethylmethacrylate beads in a continuous flow chamber. Diagn Microbiol Infect Dis. 2004;50(4):261-5.

28. Fuchs PC, Barry AL, Brown SD. In vitro bactericidal activity of daptomycin against staphylococci. J Antimicrob Chemother. 2002;49(3):467-70.

29. Mortin LI, Li T, Van Praagh AD, Zhang S, Zhang XX, Alder JD. Rapid bactericidal activity of daptomycin against methicillin-resistant and methicillin-susceptible Staphylococcus aureus peritonitis in mice as measured with bioluminescent bacteria. Antimicrob Agents Chemother. 2007;51(5):1787-94.

30. Proceedings of the International Consensus Meeting on Periprosthetic Joint Infection. https://www.efort.org/wp-content/uploads/2013/10/Philadelphia_ Consensus.pdf. Accessed 23 December 2015

31. Rouse MS, Piper KE, Jacobson M, Jacofsky DJ, Steckelberg JM, Patel R. Daptomycin treatment of Staphylococcus aureus experimental chronic osteomyelitis. J Antimicrob Chemother. 2006;57(2):301-5.

32. Hsieh PH, Huang KC, Lee PC, Lee MS. Two-stage revision of infected hip arthroplasty using an antibiotic-loaded spacer: retrospective comparison between short-term and prolonged antibiotic therapy. J Antimicrob Chemother. 2009;64(2):392-7.

33. Smith EB, Wynne R, Joshi A, Liu H, Good RP. Is it time to include vancomycin for routine perioperative antibiotic prophylaxis in total joint arthroplasty patients? J Arthroplasty. 2012;27(8 Suppl):55-60.

34. Liu C, Kakis A, Nichols A, Ries MD, Vail TP, Bozic KJ. Targeted use of vancomycin as perioperative prophylaxis reduces periprosthetic joint infection in revision TKA. Clin Orthop Relat Res. 2014;472(1):227-31. 